\title{
Research on the Difference of Physical Parameters of Concrete in Saline-alkali Environment
}

\author{
Yumao Qi, Junge Huang*, Yu Liu and Jian Pei \\ College of Urban Construction and Safety Engineering, Shanghai Institute of Technology, 201418 Shanghai, P.R.China
}

\begin{abstract}
In order to better study the corrosion of concrete in saline-alkali area, the model tests of concrete and reinforced concrete with different ions and different ion concentrations were constructed. Taking the test blocks mixed with water as the control group, the resistance and polarizability of the test block during the maintenance period were measured by rock specimen tester and rock specimen test frame. The one-way variance analysis of the measured values of each test block was carried out by spss software, investigating the difference of electrical parameters of each concrete. The results show that there is significant difference in the resistance between the clear water concrete test block and the 3\% sodium sulfate concrete test block at the level of significance $\leq 0.05$. For reinforced concrete test blocks, the resistivity difference between water test block and 3\% sodium sulfate test block and $6 \%$ sodium sulfate test block is not significant, and the resistivity difference between water test block and 15\% sodium sulfate test block and 3\% sodium chloride test block is significant. No matter concrete or reinforced concrete, the polarizability of test blocks mixed with water and test blocks mixed with each solution is significantly different. Therefore, the polarizability parameters can be used to distinguish whether the concrete is corroded by salt and alkali. It is feasible to use polarizability parameter to detect concrete erosion by salt and alkali.
\end{abstract}

\section{Introduction}

Salt and alkali are distributed in Xinjiang, northern part of China and coastal areas. Under the saline-alkali environment, concrete is vulnerable to erosion, mainly in the following ways: (1) Direct chemical erosion. (2) Base-base reaction. (3) Sulfate attack. (4) Corrosion of internal reinforcement. ${ }^{[1,2]}$ The results of salt and alkali sampling in Xinjiang show that chloride and sulfate are the main components. Xinjiang is located in the alpine and cold region, where the temperature difference between day and night is large. Under the continuous action of temperature difference, the internal cracks in the concrete structure will continue to increase ${ }^{[3]}$. In addition, the saline-alkali effect in the surrounding rock and soil and groundwater provides a channel for the ion migration in the surrounding medium of concrete, so that the corrosive medium can enter the concrete more easily. The concentration of some ions will reduce the bearing capacity of concrete, such as the formation of cement bacillus; Some ions will break the passivation film on the surface of the steel bar, causing corrosion of the steel bar and affecting the bearing capacity of the concrete structure.

In order to improve the safety of buildings, preventive measures should be taken in the early stage when harmful ions invade the concrete. Currently, there are two kinds of detection methods: chemical method and physical method. Chemical method ${ }^{[4-6]}$ is a direct detection method, that is, local sampling is carried out in sensitive parts of concrete, and harmful ion content is determined through chemical analysis. This method will cause damage to concrete structure and affect the overall performance of concrete. Physical methods include infrared thermal imaging, ultrasonic detection, resistivity, etc. The resistivity method is easily affected by the reinforcement in concrete, so it is difficult to accurately observe the variation of ions in the medium. However, the polarizability parameters are not affected by the internal reinforcement. The polarization effect inside concrete can be studied to observe the polarizability of concrete to detect the variation of ions inside concrete. Resistivity method or induced polarization method are based on the difference of physical properties of rocks and ores in the earth's crust, such as conductivity or induced polarization effect. Geological problems are solved by observing and studying the distribution law of the artificially established ground current field or excitation electric field. Therefore, in order to investigate the differences of electrical parameters of concrete specimens mixed with different salt and alkali ions, the electrical parameters such as resistivity and polarizability of each specimen during the curing period are taken as the research object, and SPSS 22 is used to judge the differences of electrical parameters of each specimen through one-way ANOVA.

\section{Test method}

\footnotetext{
* Corresponding author: Junge Huang. Email: hjg@sit.edu.cn
} 


\subsection{Test materials and mix proportion}

Ordinary portland cement with mineral composition of $\mathrm{C} 3 \mathrm{~S}, \mathrm{C} 2 \mathrm{~S}, \mathrm{C} 3 \mathrm{a}$ and $\mathrm{c} 4 \mathrm{AF}$ is used in the test. Fine aggregate is local river sand with maximum particle size of no more than $2 \mathrm{~mm}$. The coarse aggregate is crushed stone, and the part with the size of $5 \mathrm{~mm}-20 \mathrm{~mm}$ is screened out by sieve for making concrete test block. In order to reduce the experimental error, the impurities were carefully selected. Mix proportion water: cement: sand: gravel $=0.44: 1: 1.312: 2.666$, the clear water test block is mixed with tap water as the contrast test block. According to the analysis results of salt and alkali components, other test blocks were mixed with different concentrations of sodium chloride solution and sodium sulfate solution, and the concrete mix proportion design scheme is shown in Table 1. (In order to describe simply in the following table, the concrete test blocks mixed with clear water, $3 \%$ sodium sulfate solution, $6 \%$ sodium sulfate solution, $15 \%$ sodium sulfate solution and 3\% sodium chloride solution are respectively recorded as test blocks 1, 2, 3, 4 and 5. The reinforced concrete test blocks mixed with clear water, $3 \%$ sodium sulfate solution, $6 \%$ sodium sulfate solution, $15 \%$ sodium sulfate solution and 3\% sodium chloride solution are respectively recorded as test block $1 \mathrm{G}$, test block $2 \mathrm{G}$, test block $3 \mathrm{G}$, test block $4 \mathrm{G}$, test block $5 \mathrm{G}$.)

Table 1. Concrete mix design $(\mathrm{Kg})$.

\begin{tabular}{|c|c|c|c|c|c|c|c|}
\hline Sequence & Name & Solution & Solute & Water & Cement & Sand & Gravel \\
\hline 1 & test block 1 & 0.14 & 0 & 0.14 & 0.32 & 0.42 & 0.85 \\
\hline 2 & test block 2 & 0.14 & 0.0042 & 0.1358 & 0.32 & 0.42 & 0.85 \\
\hline 3 & test block 3 & 0.14 & 0.008 & 0.132 & 0.32 & 0.42 & 0.85 \\
\hline 4 & test block 4 & 0.14 & 0.021 & 0.119 & 0.32 & 0.42 & 0.85 \\
\hline 5 & test block 5 & 0.14 & 0.0042 & 0.1358 & 0.32 & 0.42 & 0.85 \\
\hline
\end{tabular}

\subsection{Specimen forming and curing}

The mixture is stirred by a mixer. First, the measured solute is slowly poured into the measured water and stirred evenly. Then, the cement, sand and gravel are added into the mixing pot for dry mixing for 30 s and evenly mixed. Finally, the solution is slowly added into the mixer for mixing.After the mixture was stirred, it was poured into a test piece of $70 \mathrm{~mm} \times 70 \mathrm{~mm} \times 90$ $\mathrm{mm}$. Then, the poured test piece is placed on the vibrating table for 5 minutes. After the vibration is completed, the excess portion is scraped off with a spatula, and the upper surface of the test piece is smoothed..The formed test piece shall be moved into the curing room for curing, and the formwork shall be removed after curing for $12 \mathrm{~h}$.

\subsection{Specimen size and observation method}

WDCB-1 rock specimen electrical tester and PDJB-1 rock specimen electrical tester developed by Chongqing Pentium numerical control technology research institute are used together to measure and store the resistivity and IP parameters of rock specimen. In order to adapt the test block model to the size of the test frame and make the measurement more accurate, the size of the test block model is set as $70 \mathrm{~mm} \times$ $70 \mathrm{~mm} \times 90 \mathrm{~mm}$. When using this device to measure the concrete test block, the current flows in or out from both ends of the test block, which can eliminate the influence of the boundary effect. After setting the relevant parameters on the instrument panel, the measurement can be started. During the measurement, the same test block is measured 10 times, and the average value is taken as the measurement data of the day. The diagram of the measuring device is as follows.

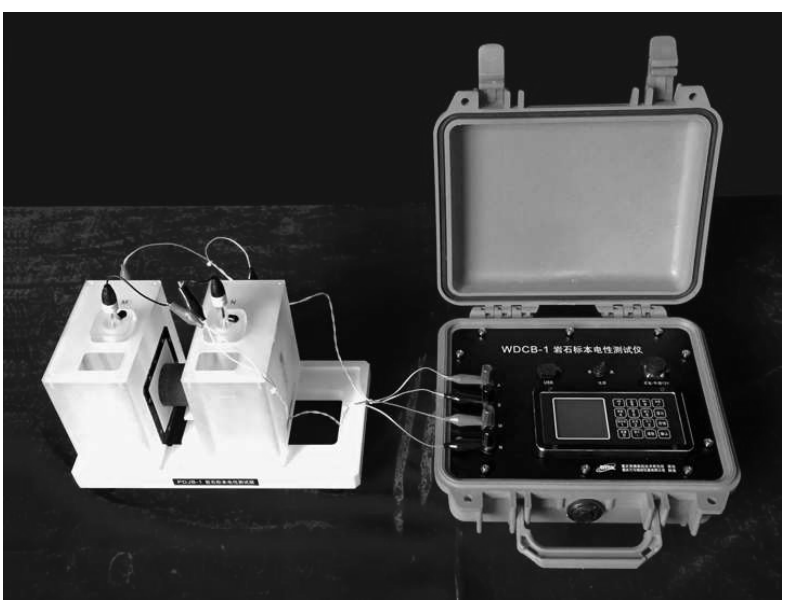

Fig. 1. Measurement device diagram.

\subsection{Analysis method}

SPSS 22 software is used to analyze the difference of the resistivity and polarizability of the test block, which can be divided into the following two cases: the difference of the electrical parameters of the test blocks mixed with the same concentration but different solute are analyzed, and the difference of the electrical parameters of the test blocks mixed with different concentration but the same solute are analyzed, so as to study the difference of the electrical parameters of each test block.

\section{The influence of salt and alkali elements on the resistivity of concrete}

The resistivity of concrete depends first on the water saturation rate of concrete pores, then on the pore 
structure of concrete, the ion concentration and temperature of pore solution. The quality of concrete (water cement ratio, setting and hardening conditions, additives, etc.) will also greatly affect the resistivity of concrete $^{[7]}$.

This section mainly compares the difference between the resistivity of as cast finish concrete test block and concrete test blocks mixed with each solution, and the difference between as cast finish reinforced concrete test block and concrete test blocks mixed with each solution, the resistivity differences of concrete mixed with $3 \%, 6 \%$ and $15 \%$ sodium sulfate solution in pairs, the resistivity differences of reinforced concrete mixed with $3 \%, 6 \%$ and $15 \%$ sodium sulfate solution in pairs, the resistivity differences of concrete mixed with $3 \%$ sodium chloride and 3\% sodium sulfate solution, and the resistivity differences of reinforced concrete mixed with 3\% sodium chloride and 3\% sodium sulfate solution. The corresponding resistivity difference analysis table is as follows.

\subsection{Analysis on the difference of the resistivity of concrete blocks mixed with different salt and alkali solutions}

Table 2. Analysis of resistivity difference of concrete blocks mixed with different salt and alkali solutions $(\Omega \cdot \mathrm{m})$.

\begin{tabular}{|c|c|c|}
\hline $\begin{array}{c}\text { Name of the } \\
\text { block }\end{array}$ & Significance & $\begin{array}{c}\text { Significance or } \\
\text { not }(\mathrm{p}<0.05)\end{array}$ \\
\hline test block 1 & \multirow{2}{*}{0.180} & \multirow{2}{*}{ No } \\
\hline test block 2 & & \\
\hline test block 1 & \multirow{2}{*}{0.003} & \multirow{2}{*}{ Yes } \\
\hline test block 3 & & \\
\hline test block 1 & \multirow{2}{*}{0.027} & \multirow{2}{*}{ Yes } \\
\hline test block 4 & & \\
\hline test block 1 & \multirow{2}{*}{0.032} & \multirow{2}{*}{ Yes } \\
\hline test block 5 & & \\
\hline test block 2 & \multirow{2}{*}{0.046} & \multirow{2}{*}{ Yes } \\
\hline test block 3 & & \\
\hline test block 3 & \multirow{2}{*}{0.457} & \multirow{2}{*}{ No } \\
\hline test block 4 & & \\
\hline test block 2 & \multirow{2}{*}{0.158} & \multirow{2}{*}{ No } \\
\hline test block 4 & & \\
\hline test block 2 & \multirow{2}{*}{0.334} & \multirow{2}{*}{ No } \\
\hline test block 5 & & \\
\hline
\end{tabular}

According to the resistivity difference of 5 concrete blocks mixed with different saline alkali solutions (Table 2), the resistivity difference between cast finish concrete block and 3\% sodium sulfate block is not significant, and the resistivity difference between cast finish concrete block and 6\% sodium sulfate, $15 \%$ sodium sulfate and $3 \%$ sodium chloride block is significant. The resistivity difference between $3 \%$ sodium sulfate block and $6 \%$ sodium sulfate block is significant, and the resistivity difference between $3 \%$ sodium sulfate block and $15 \%$ sodium sulfate block, $3 \%$ sodium chloride block is not significant. There was no significant difference in resistivity between $6 \%$ sodium sulfate block and $15 \%$ sodium sulfate block. It is precisely because the addition of ions in the test block changes the conductivity of the test block, so that the resistivity of the test block with ions is significantly different from that of the clean water test block. Moreover, the promotion of chloride ion and sulfuric acid ion on the hydration of cement will accelerate the consumption of free water in the test block and change the ion concentration in the test block, so that the resistivity values of the clean water test block and the $3 \%$ sodium chloride and $3 \%$ sodium sulfate test blocks show different differences, which can be distinguished as $3 \%$ chloride erosion or $3 \%$ sulfate erosion.

\subsection{Analysis on the difference of resistivity of reinforced concrete blocks with different saline alkali solutions}

Table 3. Resistivity difference analysis of reinforced concrete test blocks mixed with different saline and alkaline solutions $(\Omega \cdot \mathrm{m})$.

\begin{tabular}{|c|c|c|}
\hline $\begin{array}{c}\text { Name of the } \\
\text { block }\end{array}$ & Significance & $\begin{array}{c}\text { Significance or } \\
\text { not }(\mathrm{p}<0.05)\end{array}$ \\
\hline test block $1 \mathrm{G}$ & \multirow{2}{*}{0.122} & \multirow{2}{*}{ No } \\
\hline test block $2 \mathrm{G}$ & & \\
\hline test block $1 \mathrm{G}$ & \multirow{2}{*}{0.912} & \multirow{2}{*}{ No } \\
\hline test block $3 \mathrm{G}$ & & \\
\hline test block $1 \mathrm{G}$ & \multirow{2}{*}{0.010} & \multirow{2}{*}{ Yes } \\
\hline test block $4 \mathrm{G}$ & & \\
\hline test block $1 \mathrm{G}$ & \multirow{2}{*}{0.002} & \multirow{2}{*}{ Yes } \\
\hline test block 5G & & \\
\hline test block $2 \mathrm{G}$ & \multirow{2}{*}{0.128} & \multirow{2}{*}{ No } \\
\hline test block $3 \mathrm{G}$ & & \\
\hline test block 3G & \multirow{2}{*}{0.013} & \multirow{2}{*}{ Yes } \\
\hline test block $4 \mathrm{G}$ & & \\
\hline test block $2 \mathrm{G}$ & \multirow{2}{*}{0.298} & \multirow{2}{*}{ No } \\
\hline test block $4 \mathrm{G}$ & & \\
\hline test block $2 \mathrm{G}$ & \multirow{2}{*}{0.130} & \multirow{2}{*}{ No } \\
\hline test block 5G & & \\
\hline
\end{tabular}

It can be seen from the significance in Table 3 that the resistivity difference between as cast finish reinforced concrete test block and 3\% sodium sulfate, $6 \%$ sodium sulfate reinforced concrete test block is not significant, and the resistivity difference between as cast finish reinforced concrete test block and $15 \%$ sodium sulfate, $3 \%$ sodium chloride reinforced concrete test block is significant. There was no significant difference in resistivity between $3 \%$ sodium sulfate reinforced concrete and $6 \%$ sodium sulfate, $15 \%$ sodium sulfate, $3 \%$ sodium chloride reinforced concrete. The resistivity difference between $6 \%$ sodium sulfate reinforced concrete and $15 \%$ sodium sulfate reinforced concrete is significant. Because chloride ion and 
sulfuric acid ion can promote the hydration reaction of cement, accelerate the consumption of free water in the test block, change the ion concentration in the test block, so as to change the conductivity of the test block, so that the resistivity of the test block mixed with saline alkali solution and the test block with clear water presents different differences, which can be distinguished according to whether the difference is $3 \%$ chloride or $3 \%$ sulfate. At the same time, the sulfate concentration can be determined according to whether the resistivity difference between the reinforced concrete test block mixed with sodium sulfate solution and the reinforced concrete test block is significant.

\section{The influence of salt and alkali elements on the polarizability of concrete}

Generally, the polarizability is used to express the degree of induced polarization of the measured rock. It is equal to the ratio of the maximum voltage generated by the secondary electric field to the maximum voltage generated by the total electric field. The ratio eliminates the same factors, and the formula is as follows.

$$
\eta=\frac{\Delta U_{2}}{\Delta U} \times 100 \%
$$

Where $\eta$ is the polarizability, $\Delta U_{2}$ is the secondary electric field, $\Delta U$ is the sum of the primary electric field and the secondary electric field. When the polarizability parameters are used for metal ore exploration, oil exploration, seabed geological survey, etc., the polarizability value is not easily affected by the terrain, and can accurately reflect the spatial position of the polarizable body in the rock, with accurate observation. Similarly, when the polarizability method is used to observe the concrete, the influence of the reinforcement factor in the concrete is eliminated, and the change of the medium ion in the concrete can be reflected truthfully. Therefore, the change of the ion in the concrete can be detected by observing the polarizability of the concrete.

This section mainly compares the difference between the polarizability of as cast finish concrete test block and concrete test blocks mixed with each solution, and the difference between as cast finish reinforced concrete test block and concrete test blocks mixed with each solution, the polarizability differences of concrete mixed with $3 \%, 6 \%$ and $15 \%$ sodium sulfate solution in pairs, the polarizability differences of reinforced concrete mixed with $3 \%, 6 \%$ and $15 \%$ sodium sulfate solution in pairs, the polarizability differences of concrete mixed with $3 \%$ sodium chloride and $3 \%$ sodium sulfate solution, and the polarizability differences of reinforced concrete mixed with $3 \%$ sodium chloride and $3 \%$ sodium sulfate solution. The corresponding polarizability difference analysis table is as follows.

\subsection{Analysis on the difference of the polarizability of concrete blocks mixed with different salt and alkali solutions}

Table 4. Polarizability difference analysis of concrete test blocks mixed with different saline and alkaline solutions (\%).

\begin{tabular}{|c|c|c|}
\hline $\begin{array}{c}\text { Name of the } \\
\text { block }\end{array}$ & Significance & $\begin{array}{c}\text { Significance or } \\
\text { not }(p<0.05)\end{array}$ \\
\hline test block 1 & \multirow{2}{*}{0.004} & \multirow{2}{*}{ Yes } \\
\hline test block 2 & & \\
\hline test block 1 & \multirow{2}{*}{0.001} & \multirow{2}{*}{ Yes } \\
\hline test block 3 & & \\
\hline test block 1 & \multirow{2}{*}{0.000} & \multirow{2}{*}{ Yes } \\
\hline test block 4 & & \\
\hline test block 1 & \multirow{2}{*}{0.019} & \multirow{2}{*}{ Yes } \\
\hline test block 5 & & \\
\hline test block 2 & \multirow{2}{*}{0.345} & \multirow{2}{*}{ No } \\
\hline test block 3 & & \\
\hline test block 3 & \multirow{2}{*}{0.672} & \multirow{2}{*}{ No } \\
\hline test block 4 & & \\
\hline test block 2 & \multirow{2}{*}{0.191} & \multirow{2}{*}{ No } \\
\hline test block 4 & & \\
\hline test block 2 & \multirow{2}{*}{0.281} & \multirow{2}{*}{ No } \\
\hline test block 5 & & \\
\hline
\end{tabular}

From the perspective of significance (Table 4), the polarizability difference between as cast finish concrete test block and 3\% sodium sulfate concrete test block, $6 \%$ sodium sulfate concrete test block, 15\% sodium sulfate concrete test block, $3 \%$ sodium chloride concrete test block is significant, while the polarizability difference of $3 \%$ sodium sulphate concrete, $6 \%$ sodium sulphate concrete and $15 \%$ sodium sulphate concrete is not significant, but that of $3 \%$ sodium sulphate concrete and 3\% sodium chloride concrete is not significant. In fact, the polarizability of concrete will be affected by the compactness of concrete, the size of porosity, the content of conductive ions, the size and connectivity of ion channels and other factors. Adding chloride and sulfate into the above-mentioned test block will change the IP effect of the test block and make the polarizability of the test block change, which is significantly different from that of the clear water test block.

\subsection{Analysis on the difference of polarizability of reinforced concrete blocks with different saline alkali solutions}

Table 5. Polarizability difference analysis of reinforced concrete test blocks mixed with different saline and alkaline solutions (\%).

\begin{tabular}{|c|c|c|}
\hline $\begin{array}{c}\text { Name of the } \\
\text { block }\end{array}$ & Significance & $\begin{array}{c}\text { Significance or } \\
\text { not }(\mathrm{p}<0.05)\end{array}$ \\
\cline { 1 - 1 } test block 1G & 0.000 & Yes \\
\hline test block 2G & & \\
\hline
\end{tabular}




\begin{tabular}{|c|c|c|}
\hline test block $1 \mathrm{G}$ & \multirow{2}{*}{0.000} & \multirow{2}{*}{ Yes } \\
\hline test block $3 \mathrm{G}$ & & \\
\hline test block $1 \mathrm{G}$ & \multirow{2}{*}{0.000} & \multirow{2}{*}{ Yes } \\
\hline test block $4 \mathrm{G}$ & & \\
\hline test block $1 \mathrm{G}$ & \multirow{2}{*}{0.000} & \multirow{2}{*}{ Yes } \\
\hline test block $5 \mathrm{G}$ & & \\
\hline test block $2 \mathrm{G}$ & \multirow{2}{*}{0.204} & \multirow{2}{*}{ No } \\
\hline test block $3 \mathrm{G}$ & & \\
\hline test block $3 \mathrm{G}$ & \multirow{2}{*}{0.000} & \multirow{2}{*}{ Yes } \\
\hline test block $4 \mathrm{G}$ & & \\
\hline test block $2 \mathrm{G}$ & \multirow{2}{*}{0.000} & \multirow{2}{*}{ Yes } \\
\hline test block $4 \mathrm{G}$ & & \\
\hline test block $2 \mathrm{G}$ & \multirow{2}{*}{0.000} & \multirow{2}{*}{ Yes } \\
\hline test block $5 \mathrm{G}$ & & \\
\hline
\end{tabular}

From the significance point of view (Table 5), the polariability difference between test blocks mixed with water and those mixed with 3\% sodium sulfate, $6 \%$ sodium sulfate, $15 \%$ sodium sulfate and $3 \%$ sodium chloride is significant. The polarizability of $3 \%$ sodium sulfate block was significantly different from that of $15 \%$ sodium sulfate block and 3\% sodium chloride block. The polarizability difference between $3 \%$ sodium sulfate block and $6 \%$ sodium sulfate block is not significant, while the polarizability difference between $6 \%$ sodium sulfate block and $15 \%$ sodium sulfate block is significant, it is shown that the polarizability of the above test blocks is obviously different and distinguishable. By adding chloridion or sulfate ion into the test block, the IP effect of the test block was changed, and make the polarizability of the test block change, which was significantly different from that of the water test block. At the same time, because of the battery effect of reinforcement, the polarizability of each reinforced concrete test block is different from that of each concrete test block.

\section{Conclusion}

The difference analysis shows that, for concrete test blocks, the resistivity difference between water test blocks and $3 \%$ sodium sulfate test blocks is significant, and that between water test blocks and other solution test blocks is not significant. For reinforced concrete, the resistivity difference between water test block and $3 \%$ sodium sulfate test block and $6 \%$ sodium sulfate test block is not significant, and that between water test block and 15\% sodium sulfate test block and 3\% sodium chloride test block is significant.There are significant differences in the polarizability between the as cast finish concrete test block and the concrete test block mixed with each solution, and there are significant differences in the polarizability between the as cast finish concrete test block and the concrete test block mixed with each solution. Regardless of whether it is a concrete test block or a reinforced concrete test block, the difference in resistivity between the solution test blocks mixed with solution is not all significant, and the difference in polarizability between the test blocks mixed with solution is not all significant.

(2) The difference between the polarizability of the above-mentioned solution mixing test block and that of the clear water test block is significant, while the difference between the resistivity of each solution mixing test block and that of the clear water test block is not all significant. Therefore, the polarizability parameter can be used to distinguish whether the concrete is eroded by ions. It is feasible to use the polarizability parameter to detect the corrosion of concrete by salt and alkali.

\section{Acknowledgment}

Foundation item:National natural science foundation of China (41564005)

Author Introduction: Yumao Qi(1995-), Female, studying for master's degree, research direction: concrete detection and monitoring, Email: 1796018208@qq.com

* Corresponding author: Junge Huang(1966-), Male, postdoctoral, professor, research direction: Civil engineering detection and monitoring, Safety engineering. Email: hjg@sit.edu.cn

\section{References}

1. J.H Wu, Y.Z Zhao. Corrosion Monitoring/Detection of Reinforced Concrete. Journal of Corrosion and Protection, 24(10): 421427(2003).

2. B.L Jia, F.H Cao, W.J Liu, J.Q Zhang. Current Status of Electrochemical Detection of Reinforced Concrete Corrosion. Journal of Materials Science and Engineering(2010).

3. L.Y Gao, X.M Wan, X.B Ren. Study on chloride ion diffusion properties of concrete under continuous loading. Journal of Concrete and Cement Products, 192(4)21-25(2012).

4. H.Z He, M.L Shi, L.Y Cao. Design of concrete mix proportion assisted by ac impedance spectrum Journal of Shanghai Building Materials, 1:2930(2010).

5. L.N Jiang. Laboratory research on corrosion monitoring technology of metal materials in natural seawater. Master's Theses. Ocean University of China(2004).

6. L Zhang. Durability evaluation and residual life analysis of in-service reinforced concrete structures. Master's Theses. Huaqiao University(2006).

7. T Ding. Research on high-impedance and highimpedance concrete for subway. Master's Theses. Wuhan University of Technology(2008). 